\title{
A multigap RPC based detector for gamma rays
}

\author{
Georgi GEORGIEV ${ }^{a}$, Nevena ILIEVA ${ }^{b}$, Venelin KOZHUHAROV ${ }^{a}$, Iglika \\ LESSIGIARSKA $^{a}$, Leandar LITOV ${ }^{a}$, Borislav PAVLOV ${ }^{a *}$, Peicho PETKOV $^{a}$ \\ E-mail: borislav.pavlovecern.ch \\ a University of Sofia "St. Kliment Ohridski”, Sofia, Bulgaria \\ ${ }^{b}$ Institute for Nuclear Research and Nuclear Energy, Sofia, Bulgaria
}

\begin{abstract}
Transforming the resistive plate chambers from charged-particle into gamma-quanta detectors opens the way towards their application as a basic element of a hybrid imaging system, which combines positron emission tomography (PET) with magnetic resonance imaging (MRI) in a single device. We present the first results towards the development of a hybrid imaging system based on multigap glass resistive plate chambers. A GEANT4 based simulations of the efficiency of the RPC photon detectors with different converter materials and geometry were performed, leading to an optimization of the detector design. The output from these simulations together with the first prototypes are presented.
\end{abstract}

XI Workshop on Resistive Plate Chambers and Related Detectors

5-10 February, 2012

Laboratori Nazionali di Frascati dell'INFN - Frascati (Rome) - Italy

\footnotetext{
* Speaker.
} 


\section{Positron Emission Tomography}

Positron Emission Tomography (PET) is a nuclear-medicine imaging technique for registration of the whole-body distribution of positron-emitting biomarkers. Different biomarkers might be used to diagnose different diseases, but the most commonly used radiotracer in clinical PET scanning nowadays is fludeoxyglucose - a glucose analog which is labeled with fluorine-18. The injected radiolabeled biomarker is accumulated in the ill tissue. The emitted positron annihilates and produces a pair of $511 \mathrm{KeV}$ gammas, flying in opposite directions. The PET registers two gammas and reconstructs the so called Line of Response (LOR). The standard PET devices use scintillating crystals as photon detectors, coupled to photomultiplier tubes (PMT) or SiPM in some advanced designs.

The PET scan gives only the density and physiology of biomarker distribution and does not provide a clear anatomical framing. Some important hybrid PET modalities are PET/CT and MRI/PET [1]. The PET is integrated with a Computer Tomography in PET/CT and with Magnetic Resonance Imaging in PET/MRI.CT and MRI are complementary to PET, giving anatomical and morphological information to PET scan. MRI is sensitive to the soft tissue and helps to localize the human internal organs on a combined PET/MRI image. On the other hand CT is not so sensitive to the soft tissue and does not help much the PET scan in the internal organs recognition, but is very valuable in the planning of the tumor radiation therapy, due to it's ability to estimate the X-ray attenuation in the human body. The main problem in a PET/MRI system is that the photomultiplier tubes, traditionally used to detect the scintillations, are sensitive to magnetic field. The successful detector in a hybrid PET/MRI should not be affected by strong magnetic field and that is why in the modern systems SiPMs and dSiPMs are used.

Sub-millimeter spatial resolution is a must for mammography PET [2] and small animal PET [3] systems. The small animal PET systems are used by pharmaceutical companies for pharmacokinetic research. In such a system the Compton scattering can be neglected but the position resolution is of primary importance.

The intrinsic properties of the annihilation process, like the finite positron range in the tissue and photons non-collinearity due to the residual positron momentum, limit the PET accuracy to few millimeters. The scintillating crystals detectors used in the commercial PET systems impose additional limits to PET resolution and the purpose of our study is to show that the RPC detectors are capable to overcome these additional limitations. Few processes in the detector are responsible for physical limitations of PET:

- Random coincidence. If gammas from two different annihilations are detected by PET, spurious line will be reconstructed. Increasing the detector time resolution will decrease the probability for simultaneous detection of two gammas from different annihilations (figure 11a).

- Compton scattering. The gamma may scatter in the medium. That leads to wrong LOR reconstruction. The detector should suppress the Compton scattered gammas (figure 1 $\mathrm{b} b$ ). 

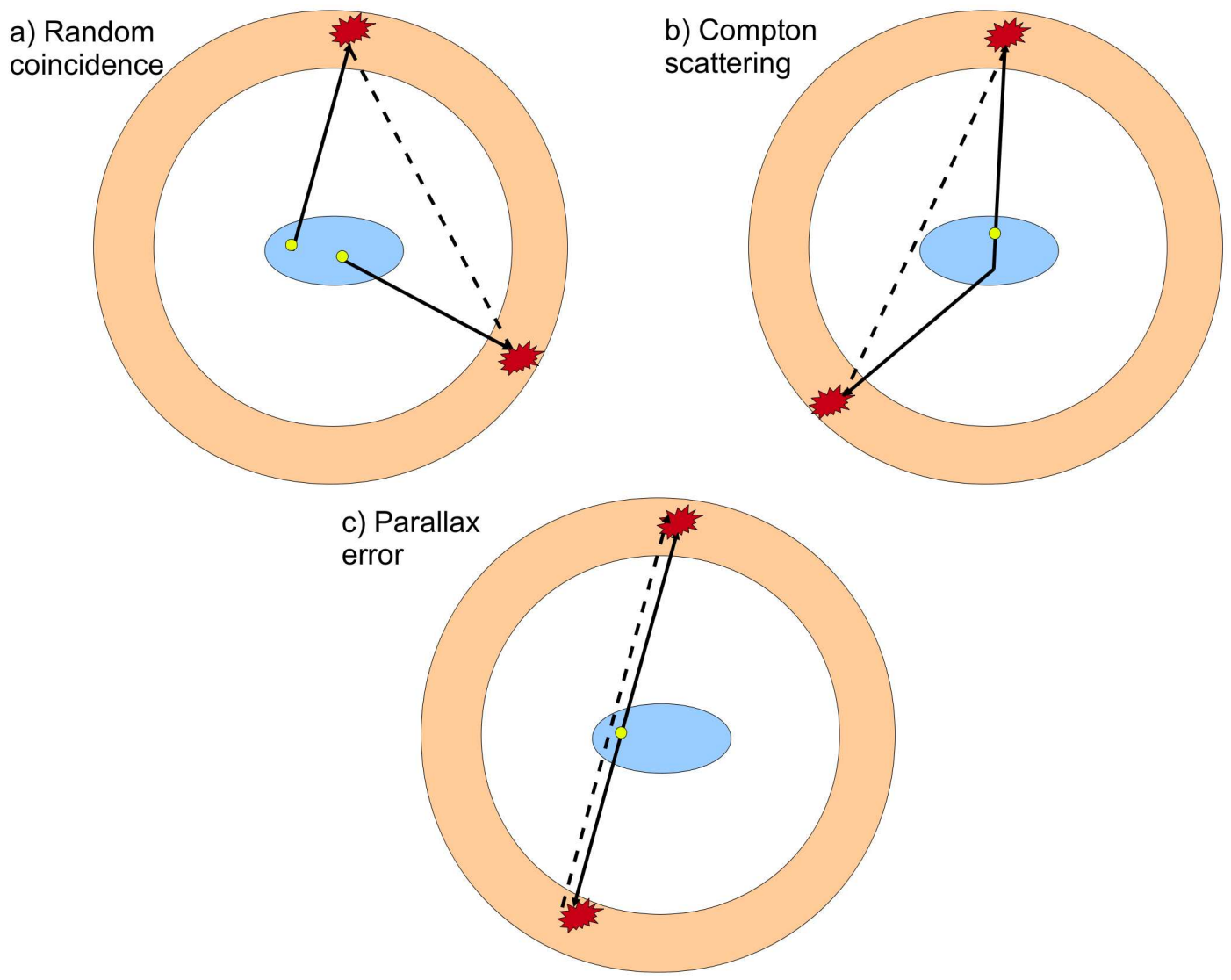

Figure 1: Processes that degrade PET resolution : a) random coincidence; b) Compton scattering; c) parallax error

The standard solution is to measure the photon energy and to perform a cut. Thus, a good detector energy resolution is needed.

- Parallax error depends on the detector spatial resolution (figure 1 $1 \mathrm{c}$ ). Detectors with high spatial resolution are needed.

\section{RPC in a PET system}

The RPCs have some advantages over scintillating crystals and definitely might overcome

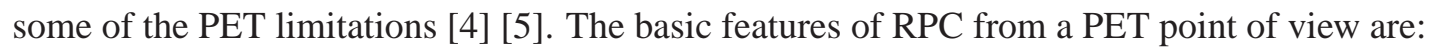

- Unique time resolution: 20 ps time resolution for charged particles is reported in the literature [6];

- Extremely good position resolution. Few hundred microns are not a challenge for RPC detector technology;

- No parallax error; 
- Not affected by strong magnetic fields, thus can be used together with a MRI;

- Effective Compton suppression without energy measurement;

- Much cheaper than crystals;

- Easy to build large area RPC [7] [8];

The excellent RPC timing resolution opens the possibility to measure photons Time of Flight (ToF). The ToF information constrains the positron annihilation position to a few millimeters region on the LOR, thus enhancing the image reconstruction and essentially reducing patient's dose ${ }^{1}$ - up to ten times without image quality loss, according to the esteems. The ToF information helps Compton suppression. A good ToF information will reduce also the acquisition time and will open the way to new PET medical applications in cardiology and neurology. The RPCs with their exceptional time resolution are suitable for ToF PET.

The good position resolution enables mammography PET and small animal PET applications. The RPCs are cheap and large area detectors are easy to be build. RPC might be used in a whole body PET system, greatly incising the field of view (FOV), decreasing the acquisition time and the patient's dose.

\section{RPC design and Simulations}

The two main goals of the successful RPC-PET design are detector efficiency increase for $511 \mathrm{KeV}$ photons and suppression or rejection of Compton-scattered photons. We suggest a modification of the RPCs with increased efficiency for $511 \mathrm{KeV}$ gammas. The key idea is to choose appropriate materials for one or both of the electrodes, thus transforming them into a gamma-toelectron converters. The schematic design is shown on the Fig. 2. The gamma quanta from the positron annihilation interact with the converter medium, the ejected electrons pass to the gas gap and start an avalanche. We investigated three different detector designs.

One of the considered designs is Gas - Converter (GC) design. The direct contact between the converter and the gas apparently facilitates the propagation of the emitted electrons into the gas gap. The simulated GC design includes a $300 \mu \mathrm{m}$ gas gap and $2 \mathrm{~mm}$ glass plate (Fig. 2 and different converter materials and thicknesses.

We simulated 5 different high $\mathrm{Z}$ converting materials - Bismuth (Bi), Lead (Pb), Gold ( $\mathrm{Au})$, Tungsten (W) and Platinum (Pt), in direct contact with the gas volume. We are aware of the possible problems arising from the direct contact between the metal electrode and the gas. One of the concerns is the aging. Another concern is that an excellent conductor like Gold or Platinum might trigger discharges in the RPC. But it seems not to be the case, because only the resistivity of the anode is crucial - the Hybrid Parallel Plate Counter operated with a Gold electrode as a cathode [9]. Moreover one can keep the converting properties of the material and greatly change the electrical properties. For example Lead has atomic number $\mathrm{Z}=82$ and density $\rho=11.34 \mathrm{~g} . \mathrm{cm}^{-3}$, Lead

\footnotetext{
${ }^{1}$ the typical PET dose is approximately $8 \mathrm{mSv}$, according to IAEA
} 

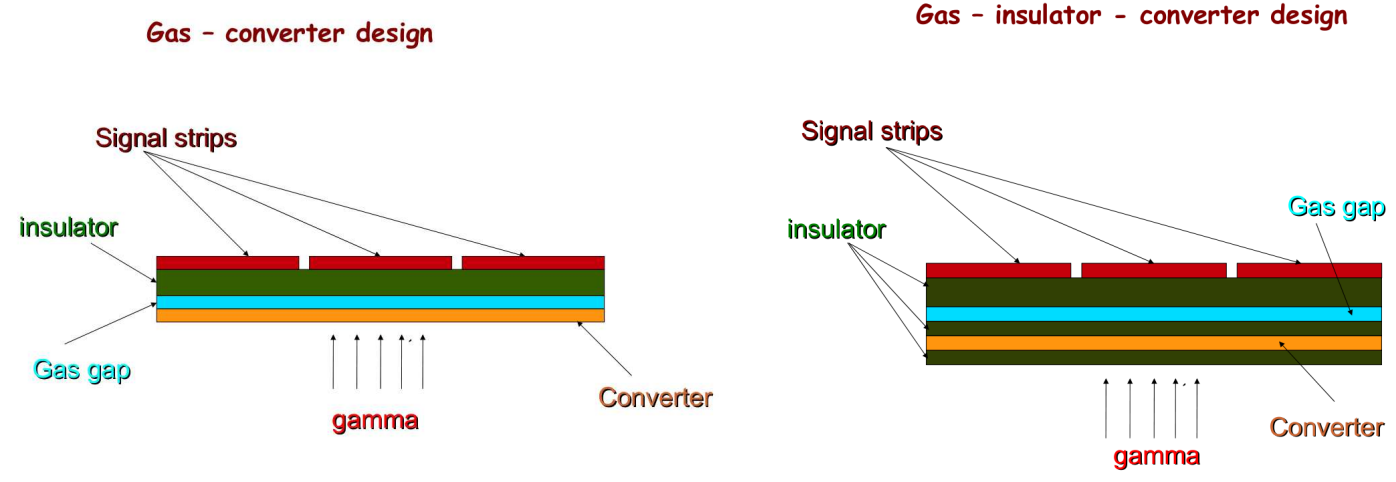

Multi-gap GIC design

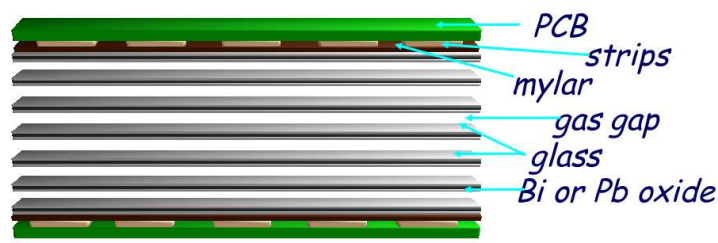

Figure 2: Gas-Converter design (upper left); Gas-Insulator-Converter design (upper right); Multigap GasInsulator-Converter design (lower center)

Oxide $(\mathrm{PbO})$ has effective atomic number $Z_{\text {eff }} \approx 79.4$ and density $\rho=9.53 \mathrm{~g} . \mathrm{cm}^{-3}$. Lead is a poor conductor, but Lead Oxide is an excellent insulator.

The investigated parameter is the electron yield in the gas. The electron yield is the probability to have an electron ejected in the gas, after the photon interaction with the converter. Electron yield depends on two processes - photon interactions in the converter, and electron propagation through the converter to the gas. The conversion probability increases with the converter thickness, but on the other hand the electron has a finite range in the converter and thus a maximal yield can be defined.

Different converter thicknesses from 1 to $100 \mu \mathrm{m}$ were simulated. The yield saturates at about $40 \mu \mathrm{m}$, giving the maximal yield for each material. The maximal yield ranges from 0.30 to $0.38 \%$ and is highest for Bismuth $(0.382 \pm 0.009 \%)$, followed by Lead $(0.380 \pm 0.007 \%)$. Unfortunately, the electron yield is higher for the low energy (Compton scattered) electrons than for $511 \mathrm{KeV}$ photons. The result clearly disproves the intuitive gas-converter design.

Next considered design is Gas-Insulator-Converter design. A Bismuth converter sandwiched between two glass plates is used as a cathode in that RPC design (Fig. Q). The clear design advantage is the avoidance of the direct contact between the gas and the converting material. The glass surface is highly resistive and smooth and thus prevents discharges. Furthermore the glass is traditionally used in the glass RPCs and it's aging is well studied [10]. 
We simulated different converter thicknesses ranging from 1 to $100 \mu \mathrm{m}$ for 5 different photon energies $(102,204,307,409$ and $511 \mathrm{KeV})$. For $200 \mu \mathrm{m}$ glass, the electron yield for $511 \mathrm{KeV}$ photons saturates at converter thickness of about $40 \mu \mathrm{m}$ and is $(0.228 \pm 0.020) \%$. The yield is $40 \%$ lower in comparison to previous design. But the electron yield for $307 \mathrm{KeV}$ photons drops to $(0.045 \pm 0.008) \%$, which is about $90 \%$ lower compared to the direct contact construction, so a clear gain in Compton suppression is evident. The insulator thickness is also important. At a fixed converter thickness $(40 \mu \mathrm{m})$ the electron yield for $511 \mathrm{KeV}$ decrease linearly with the increase of the insulator thickness, but the electron yield for the Compton-scattered photons drops rapidly after $50 \mu \mathrm{m}$ insulator thickness. The result points to an optimal converter construction of $50 \mu \mathrm{m}$ Bismuth converter and $50 \mu \mathrm{m}$ glass insulator.

We simulated a stack of 100 gas gaps, formed by glass $(50 \mu \mathrm{m})$-Bismuth $(50 \mu \mathrm{m})$-glass $(50 \mu \mathrm{m})$ plates. The gas mixture is $85 \% \mathrm{C}_{2} \mathrm{H}_{2} \mathrm{~F}_{4}, 5 \% \mathrm{i}-\mathrm{C}_{4} \mathrm{H}_{10}, 10 \% \mathrm{SF}_{6}$, but it is only considered for material budged. The avalanche development in the gas is not simulated in the study. The electron yield from the simulation is $(23.8 \pm 0.4) \%$ which is comparable with the crystal PET detectors. The highest yield is observed for $511 \mathrm{KeV}$ photons, the sensitivity for photons with $307 \mathrm{KeV}$ is two times smaller.

The convolution of a realistic human body photon spectrum and detector response shows that about $86 \%$ of the registered in the PET process photons are with energies above $380 \mathrm{KeV}$ and are suitable for PET reconstruction [11].

\section{RPC prototypes}

The simulation results are encouraging and we adopted a multi-gap Converter-Insulator-Gas design in our prototypes. The basic module is a six-gap glass RPC with readout strips on he both sides. Fishing lines are used for spacers. Graphite paint is used to form the high voltages electrodes on the outermost glasses. The high voltage electrodes are insulated from the readout strips by a Mylar foil. The copper readout strips are implemented on PCB boards. Twenty independent six-gap RPCs should be stacked one on top of the other to form 120 gas gaps supermodule.

We constructed several small RPCs and one full scale RPC. The design parameters are listed in the Table.

\begin{tabular}{|l|c|c|}
\hline & small prototype & full scale prototype \\
\hline number of gaps & 6 & 6 \\
gas gap & $200 \mu \mathrm{m}$ & $200 \mu \mathrm{m}$ \\
glass thickness & $150 \mu \mathrm{m}$ & $100 \mu \mathrm{m}$ \\
dimensions & $120 \mathrm{~mm} \times 70 \mathrm{~mm}$ & $350 \mathrm{~mm} \times 70 \mathrm{~mm}$ \\
\hline
\end{tabular}

We tried different techniques and materials, for example Lead containing resistive paint, to form the converting layer between the two glasses. 


\section{Conclusions}

We performed a model investigations towards the design of an RPC-based PET detector. The intuitive direct contact between converter and the gas does not provide sufficient Compton suppression. The proposed multigap gas-converter-insulator design ensures high enough electron yield for $511 \mathrm{KeV}$ photons with a sufficient Compton suppression factor. For 100 gaps stack made out of glass-bismuth-glass sandwich type converting resistive plates the electron yield is $24 \%$ for 511 $\mathrm{KeV}$ photons and the sensitivity for $511 \mathrm{KeV}$ photons is twice higher than the sensitivity for 307 $\mathrm{KeV}$ photons. The detailed simulation shows that about $86 \%$ of the registered in the PET process photons are with energies above $380 \mathrm{KeV}$ and are suitable for PET reconstruction.

\section{Acknowledgments}

The work is supported by National Science Fund (Bulgaria) under contract number DO $02-$ 183/2008. We acknowledge the useful comments and discussions with Crispin Williams, Paolo Vitulo, Sergio Ratti and Marec Palka.

\section{References}

[1] B. Pichler et al, Multimodal imaging approaches: PET/CT and PET/MRI, Handb Exp Pharmacol. 185 (2008) 109-32

[2] R. Raylman et al, The positron emission mammography/tomography breast imaging and biopsy system (PEM/PET): design, construction and phantom-based measurements, Phys. Med. Biol. 53 (2008) 637 [doi:10.1088/0031-9155/53/3/009]

[3] E. Visser et al, Spatial resolution and sensitivity of the Inveon small-animal PET scanner, J Nucl Med 50 (2009) 139-47

[4] A. Bianco et al, RPC-PET: A New Very High Resolution PET Technology, IEEE Transactions on Nuclear Science, 53, (2006) 2489 - 2494

[5] G. Belli et al, RPC: from High Energy Physics to Positron Emission Tomography, J. Phys.: Conf. Ser. 41 (2006) 555 [doi:10.1088/1742-6596/41/1/068]

[6] C. Williams et al, A 20 ps timing device-A Multigap Resistive Plate Chamber with 24 gas gaps, Nucl. Instr. Meth. A 594 (2008) 39-43 [doi:10.1016/j.nima.2008.06.013]

[7] M. Abbrescia et al, Cosmic ray tests of double-gap resistive plate chambers for the CMS experiment, Nucl. Instrum. Meth. A 550 (2005) 116-126 [doi:10.1016/j.nima.2005.06.074]

[8] G. Chiodini et al, RPC cosmic ray tests in the ATLAS experiment, Nucl. Instrum. Meth. A $\mathbf{5 8 1}$ (2007) 213-216 [doi:10.1016/j.nima.2007.07.080]

[9] C. D'Ambrosio et al, A Hybrid Parallel Plate gas Counter for medical imaging, Nucl. Instrum. Meth. A 572 (2007) 244-245 [doi:10.1016/j.nima.2006.10.193]

[10] C. Gustavino et al, Ageing and recovering of glass RPC, Nucl. Instrum. Meth. A 533 (2004) 116-120 [doi:10.1016/j.nima.2004.07.012]

[11] N. Ilieva et al, Development of a Novel PET Imaging System, Based on Resistive-PlateChambers (RPC), AIP Conf. Proc. 1203 (2009) 820-825 [doi:10.1063/1.3322562] 